\title{
Oral contraceptives and VTE risk in PCOS
}

Use of combined oral contraceptive therapy increases risk of venous thromboembolism (VTE) in women with polycystic ovary syndrome (PCOS), show the results of a new population-based cohort study in the USA published in the Canadian Medical Association Journal.

Existing PCOS guidelines do not provide definitive information about cardiovascular end points, comments study researcher Steven Bird, although recent work has indicated that women with this syndrome are at increased risk of VTE. Use of oral contraceptives is associated with an increased risk of VTE in the general population, but whether these drugs exacerbate VTE risk in patients with PCOS was not clear.

Bird et al. examined the IMS LifeLink Health Plan Claims database to identify 43,506 new users of oral contraceptives with PCOS, who were matched with a control group of 43,506 new users of oral contraceptives without PCOS. A random sample of 2 million US women with and without PCOS who did not take oral contraceptives was also assessed to determine baseline VTE risk.

PCOS and use of oral contraceptives were shown to be independent risk factors for VTE. Among women not receiving oral contraceptives, the risk of VTE was 1.5-fold increased in those with PCOS compared with those without PCOS. Use of oral contraceptives by women with PCOS further increased their risk of VTE, as these women had a twofold increased risk compared with women without PCOS taking oral contraceptives.

"Although the risk for the development of blood clots is small, prescribers should consider the increased risk in women with PCOS who are prescribed contraceptive therapy," says Bird. "Further research should be conducted to address VTE risk by severity of PCOS," he adds.

Joana Osório

Original article Bird, S. T. et al. Risk of venous thromboembolism in women with polycystic ovary syndrome: a population-based matched cohort analysis. CMAJ doi:10.1503/cmaj.120677 